population. To date, only a few reports on behavioural risk correlates and infection history of male clients have been documented in the United States, mainly drawn from populations in central cities and at high-risk for HIV. This study examines STIs/HIV behavioural risks and screening history correlates of male clients in the general population and whether the prevalence of male clients differs by urbanisation level.

Methods Data from the 1999-2000 National STD and Behavioral Measurement Study, a cross-sectional telephone-based probability sample of the non-institutionalised population aged 18 to 45 years old, was linked to the National Center Health Statistics Urban Rural Classification Scheme for Counties. The analysis is restricted to men aged 26 to 45 years old $(\mathrm{N}=469)$. F-statistic that accounted for survey design effects and Poisson regression for weighted data were used.

Results The overall prevalence of clients was 14.5 (95\% CI 11.518.1) and did not statistically differ between men residing in central cities of large metropolitan areas (MA) and men residing in large metropolitan suburbs, MAs of less than one million people, or nonmetropolitan subdivisions $(\mathrm{P}=0.5655)$. Compared to non-clients, clients had a significantly higher prevalence of an array of other high-risk behaviours in their lifetime and more recently including history of same-sex partners (Adjusted prevalence ratio (Adj. $P R)=2.5$, 95\% CI, 1.5-4.1), sold sex (Adj.PR = 3.6, 95\% CI, 2.2-6.1), multiple partners in the past year (Adj.PR $=2.6,95 \% \mathrm{CI}, 1.6-4.0$ ), as well as a history of STIs (Adj.PR $=2.595 \%$ CI 1.5-4.4).

Conclusion Male clients are ubiquitous. The risk-taking behaviour of clients in the general population is important to develop more comprehensive prevention measures for STIs/HIV.

\section{P3.302 SEXUAL HEALTH EXPERIENCE AND KNOWLEDGE OF HUMAN PAPILLOMAVIRUS (HPV) AND CHLAMYDIA IN YOUNG WOMEN RECRUITED VIA SOCIAL NETWORKING SITES}

doi:10.1136/sextrans-2013-051184.0757

'S M Garland, ${ }^{2} \mathrm{~B}$ Gunasekaran, ${ }^{3} \mathrm{~N}$ Ahmed, ${ }^{4} \mathrm{Y}$ Fenner, ${ }^{1} Y$ Jayasinghe, ${ }^{5} \mathrm{E}$ Moore, ${ }^{4} \mathrm{~A}$ Fletcher, 'S Tabrizi, ${ }^{6} \mathrm{~J}$ Wark. 'Dept of Microbiology \& Infectious Diseases The Royal Womens Hospital, Dept of Obstetrics \& Gynaecology, University of Melbourne \& Dept of Microbiology, The Royal Childrens Hospital \& Murdoch Childrens Research Institute, Parkville, Australia; ${ }^{2}$ Dept of Microbiology \& Infectious Diseases The Royal Womens Hospital, Dept of Microbiology The Royal Childrens Hospital \& Murdoch Childrens Researh Institute, Parkville, Australia; ${ }^{3}$ Dept of Microbiology \& Infectious Diseases The Royal Womens Hospital, Dept of Obstetrics \& Gynaecology, University of Melbourne, Parkville, Australia; ${ }^{4}$ Dept of Microbiology \& Infectious Diseases The Royal Womens Hospital, Dept of Microbiology, The Royal Childrens Hospital \& Murdoch Childrens Research Institute, Parkville, Australia; ${ }^{5}$ Dept of Microbiology \& Infectious Diseases The Royal Womens Hospital, Dept of Microbiology, The Royal Childrens Hospital \& Murdoch Childrens Research Institute, Parkville, Australia; ${ }^{6}$ Department of Medicine, Royal Melbourne Hospital, Department of Medicine, University of Melbourne, Parkville, Australia

Background Monitoring sexual health of young Australian women is a major research priority since introduction of the federally funded HPV vaccination programme, plus screening programmes for chlamydia. Social networking sites (SNS), commonly used by young people, present an opportunity for innovative recruitment modalities. We assessed young women's knowledge and experience of chlamydia, HPV, HPV vaccines and cervical cytology (Pap smears) utilising Facebook

Method This was part of a feasibility study assessing use of Facebook to recruit subjects for a larger prospective health study, the Young Female Health Initiative (YFHI). Women 16 to 25 years, from Victoria, Australia were eligible to participate. An advertisement was placed on Facebook for 6 months and visible to a random sample of eligible women. Women clicking on the advertisement were redirected to our website, then contacted and asked to complete a survey at the YFHI study centre, or the questionnaire online. The survey contained demographic questions, plus sexual health questions.

Results We enrolled 426 respondents, of whom 278 completed the survey $(50 \%$ study centre, $50 \%$ online). Respondents' socioeconomic and geographical distribution (urban, regional, rural) were representative of the target population; those $>18$ years were more likely to enrol than $16-17$ year olds ( $p<0.05$ ). Overall, $76 \%$ had been sexually active, median age of coitarche was 16.9 years [CI 16.6-17.2], 63\% had heard of HPV: of these, 73\% knew HPV is sexually acquired and $94 \%$ that it causes cancer. $78 \%$ had heard of chlamydia: those who were sexually active were more likely to know of chlamydia than were virgins $(p<0.01)$, while $63 \%$ knew it could cause chronic pelvic pain, and $86 \%$ that it could cause infertility. This recruitment method also was cost-effective (\$USD 20 per compliant participant).

Conclusions SNS is an effective recruitment strategy to engage young women in sexual health research

\section{P3.303 HIV PREVALENCE AND ASSOCIATED RISK FACTORS AMONG UNIVERSITY STUDENTS IN EASTERN ETHIOPIA, 2009}

doi:10.1136/sextrans-2013-051184.0758

\section{B Mengistu. NASTAD, Addis Abeba, Ethiopia}

Background The students in University enjoy the freedom of living outside the guidance of their parents; if this is not managed properly it increases the likelihood to have of risky sexual behaviour and HIV infection. It is imperative to monitor HIV among young people in universities for proper and timely action.

Objective This research objective was to determine the prevalence of HIV infection among Dire Dawa university students and identify risk factors for HIV sero-positivity.

Method A cross sectional study with internal comparison was conducted among students of Dire Dawa. A self administrated questionnaire was used to gather relevant information and blood test was conducted for HIV antibody. The two information were linked anonymously. Qualitative data was collected using Focus group discussion.

Result The prevalence of HIV was $2.5 \%$ (95\% CI 1.5, 3.5) (2.3\% for female and $2.7 \%$ for male) with no significant difference between male and female. The students having sexual affair with same sex or both sexes were significantly 10.45 times $(95 \%$ CI $1.93,56.41)$ to be exposed to HIV than those having sexual affairs with only opposite sex. Having non-regular partner is significantly associated with HIV positivity among sexually active $(\mathrm{OR}=6.35$ and $95 \%$ CI $2.2,18.3)$. Drinking alcohol and chewing chat was a risk factor for commencing sexual intercourse among male with $(\mathrm{OR}=2.73$ and $95 \% \mathrm{CI}$ $1.72,4.33)$ and $(\mathrm{OR}=2.02$ and $95 \%$ CI $1.19,3.42)$ respectively.

Conclusion The students in the university are at risk of HIV infection, it is necessary to target them earlier in the high school and later when joining college by building their knowledge and skill to avoid risky sexual behaviour.

\section{P3.304 WITHDRAWN BY AUTHOR}

\section{P3.305 VULNERABILITY OF SEX WORKERS (SWS) AND THEIR PARTICULAR NEEDS FOR HIV/STI PREVENTION, DIAGNOSIS, AND TREATMENT: BORDERNETWORK RESEARCH FINDINGS AND RECOMMENDATIONS FOR COMPREHENSIVE SEXUAL HEALTH RESPONSE}

doi:10.1136/sextrans-2013-051184.0759

'E Steffan, 'T Arsova Netzelmann, ${ }^{2} \mathrm{~L}$ Lõhmus, ${ }^{3} \mathrm{~J}$ Kalikov, ${ }^{4} \mathrm{~A}$ Karnite, ${ }^{5} \mathrm{~A}$ Leffers, ${ }^{6} \mathrm{~B}$ Kucharova, ${ }^{7} \mathrm{R}$ Dimitrova, ${ }^{8} \mathrm{C}$ Fierbinteanu. ${ }^{1} \mathrm{SPI}$ Forschung gGmbH, Berlin, Germany ${ }^{2}$ National Institute for Health Development, Tallinn, Estonia; ${ }^{3} \mathrm{AIDS}$ Information and 
Support Centre, Tallinn, Estonia; 'Latvia's Association for Family Planning and Sexual Health, Riga, Latvia; ${ }^{5}$ Aids Hilfe Potsdam, Potsdam, Germany; ${ }^{6}$ C.A. Prima, Bratislava, Slovakia; ${ }^{7}$ Health and Social Development Foundation, Sofia, Bulgaria; ${ }^{8}$ Romanian Associations against AIDS, Bucharest, Romania

Background Prostitution is a frequent and often controversial discussion topic at expert and political levels in Europe. Still evidencebased knowledge of sex work in general, and the situation of the SWs in particular, is scarce. BORDERNETwork addressed these gaps with an integrated bio-behavioural survey in seven EU countries. It compiled contextualised knowledge on the health and social situations of SWs, detected prevalence of and vulnerability to HIV/STIs, and formulated prevention practise recommendations.

Methods Behavioural and epidemiological data was collected between March 2011 and February 2012 through qualitative faceto-face interviews (an 85-item questionnaire) and blood screening tests (HIV, Syphilis, HCV, and HBV) among 956 SWs (respondentsand service-driven sample) in six capital cities and a border area.

Results The findings outlined multiple risks: About 38\% of the SWs had experience injecting drugs, $60 \%$ lacked health insurance. $59 \%$ had had an HIV test in the past year, but STI/sexual health services are hardly utilised. $77.1 \%$ had not attended an STI-specialist and $51.1 \%$ had not visited a gynaecologist in the past year. The prevalence for HIV was $4.6 \%$, for Syphilis - $4.5 \%$, for Hepatitis B $6.2 \%$, and for Hepatitis C - $24 \%$ (over $90 \%$ among PWID). Risk predictors linked in particular to increased prevalence of Syphilis, HBV, and HCV were drug and alcohol use before/during sex work and inconsistent condom use during oral/vaginal sex with clients.

Conclusions The behavioural and social determinants of risk, including precarious living conditions and stigma, multiply the vulnerability of SWs impeding their access to health and social care. To that end policy regulations should endorse the creation of structures for early and easy access to health services. An adequate health care provision package (incl. sexual health) should be envisaged including those SWs lacking health, social insurance and legal status in the country of stay also.

\section{P3.306 POPULATION CHANGE AND MALE CLIENTS OF SEX WORK IN U.S. METROPOLITAN AREAS}

doi:10.1136/sextrans-2013-051184.0760

M A Villarroel. Johns Hopkins Bloomberg School of Public Health, Baltimore, MD, United States

Background Rate of population change gives an indication of the average economic prosperity in a geographic area that reflects changing conditions to its physical and social environment which may influence the STIs risk behaviours found. Men who buy sex from sex workers (clients) are at high-risk of STIs and key in the spread infection to the general population. This study analyses census data matched with survey data to examine the association between population change in U.S. metropolitan counties with clients.

Methods A sample of 385 metropolitan men embedded in a 19992000 national probability telephone survey was linked to 1990 and 2000 U.S. Census county population counts. County population change between 1990 and 2000 was measured categorically and as a transformed continuous variable to account for negative growth and its skewed distribution. Poisson regression was used to obtain prevalence ratio (PR).

Results The sample was $73 \%$ non-Hispanic white, median age of 36 years, $77 \%$ were currently married or living with a partner and $39 \%$ had completed at least four years of college. The prevalence of clients was $14.8 \%$ (CI $11.5-18.9 \%$ ), differing significantly by the population relative change of the county of residence $(\mathrm{P}<0.05)$. The lowest and highest prevalence of clients was observed among men residing in counties that loss population $(6.0 \%$ (CI, 2.0-17.2) and grew rapidly above the national average $(21.4 \%$, CI, $13.5 \%-32.3 \%)$.
Using the transformed continuous measure for population change, there was a statistically significant increase in the ratio of male clients with a unit increase in population growth above the national average (unadjusted PR, 0.2, CI, 0.1-0.5, P < 0.010).

Conclusion The relationship between rate of urbanisation in metropolitan areas and male clients provides support for demographic changes influencing level of STI risks in the population and may contribute to development of comprehensive STI prevention activities.

\section{P3.307 POPULATION GROWTH WITHIN REGIONAL PATTERN OF POPULATION CHANGE AND MALE CLIENTS OF SEX WORKERS IN U.S. METROPOLITAN AREAS}

doi:10.1136/sextrans-2013-051184.0761

M A Villarroel. Johns Hopkins Bloomberg School of Public Health, Baltimore, MD, United States

Background Male clients of sex workers are at high-risk of HIV and are a key group in the spread of sexually transmitted infections to the general population. Places undergoing population growth are altering their economic, physical and social environments which may influence the pattern of sexual risk behaviour. However population growth is not uniform within or between U.S. metropolitan areas and there is an interrelated pattern of growth within a metropolitan area. This study examined the association of county population growth that was slower versus similar and faster than its own metropolitan area's pace of population change with male clients in a sample of men $(N=303)$ residing in U.S. metropolitan areas embedded in a 1999-2000 national probability telephone survey of STD behavioural measurements.

Methods Population counts for non-institutionalised population at the county level were obtained from the 1990 and 2000 U.S. Census and the total household population in each metropolitan area was calculated by adding the household population of all its component counties. County population change relative to its metropolitan area was quantified using location quotient. Poisson regression was used to obtain prevalence ratio (PR).

Results The prevalence of male clients was $17.5 \%$. In unadjusted analysis, there was no statistically significant relationship between clients and whether the county of residence experienced a rate of growth that was slower versus similar ( $\mathrm{PR}=1.79,95 \% \mathrm{CI} 0.9-3.3, \mathrm{P}>0.05)$ or faster ( $\mathrm{PR}=1.4,95 \%$ CI $0.7-2.9, \mathrm{P}>0.05)$ to its overall metropolitan area. After adjusting for county-level demographical differences at the starting period, clients were however more likely to reside in counties that experienced a similar ( $\mathrm{PR}=2.33,95 \% \mathrm{CI} 1.1-5.0, \mathrm{P}<0.05)$ or a faster $(\mathrm{PR}=2.5,95 \% \mathrm{CI} 1.1-5.9, \mathrm{P}<0.05)$ pace of growth.

Discussion Male clients of sex workers may not be similarly distributed across metropolitan areas and demographic changes may influence patterns of STI risks.

\section{P3.308 THE CHANGING MALE SEX WORKER POPULATION IN LONDON (2002 - 2012)}

doi:10.1136/sextrans-2013-051184.0762

R Malek, L Mulka, G King, A Scott, D Wilkinson. Imperial College Healthcare NHS Trust, London, UK

Background With freedom of movement across European borders, increasing globalisation and emergence of new major economies, the UK has seen significant changes to the composition of nationalities migrating to the UK over the last ten years. In turn this has changed the working population of the UK. The objective of this study was to investigate the changes in nationalities of male sex workers (MSW) attending a dedicated clinic for MSW in London over the last decade.

Methods Clinic records for MSW attending a dedicated clinic in Central London were reviewed (1/1/2002 - 31/12/2002 and 